\title{
ANTITONICITY OF THE INVERSE FOR SELFADJOINT MATRICES, OPERATORS, AND RELATIONS
}

\author{
JUSSI BEHRNDT, SEPPO HASSI, HENDRIK WIETSMA, AND HENK DE SNOO
}

(Communicated by Marius Junge)

\begin{abstract}
Let $H_{1}$ and $H_{2}$ be selfadjoint operators or relations (multivalued operators) acting on a separable Hilbert space and assume that the inequality $H_{1} \leq H_{2}$ holds. Then the validity of the inequalities $-H_{1}^{-1} \leq-H_{2}^{-1}$ and $H_{2}^{-1} \leq H_{1}^{-1}$ is characterized in terms of the inertia of $H_{1}$ and $H_{2}$. Such results are known for matrices and boundedly invertible operators. In the present paper those results are extended to selfadjoint, in general unbounded, not necessarily boundedly invertible, operators and, more generally, for selfadjoint relations in separable Hilbert spaces.
\end{abstract}

\section{INTRODUCTION}

Let $H_{1}$ and $H_{2}$ be selfadjoint matrices, operators, or relations (multivalued operators) in a separable Hilbert space, which is not necessarily finite-dimensional. This paper is concerned with a question which goes back to $\mathrm{K}$. Löwner: what are the implications of the inequality $H_{1} \leq H_{2}$ for the inverses of $H_{1}$ and $H_{2}$ (cf. [1, 16])? In the literature such results are often formulated as antitonicity results; see e.g. 4, 11, 17, 19.

Here specific conditions are investigated under which the implication

$$
H_{1} \leq H_{2} \quad \Rightarrow \quad H_{2}^{-1} \leq H_{1}^{-1}
$$

is true. Of course, the above implication does not hold in general; a simple counterexample is $H_{1}=-I$ and $H_{2}=I$. In the finite-dimensional setting necessary and sufficient conditions for the implication in (1.1) to hold are given by the following antitonicity theorem; see [4,17. Recall that the inertia of the selfadjoint matrix $H_{i}, i=1,2$, is the ordered triplet, $\mathrm{i}\left(H_{i}\right)=\left\{\mathrm{i}_{i}^{+}, \mathrm{i}_{i}^{-}, \mathrm{i}_{i}^{0}\right\}$, of the numbers of positive, negative, and zero eigenvalues of $H_{i}$ (counting multiplicities).

Theorem 1.1. Let $H_{1}$ and $H_{2}$ be invertible selfadjoint matrices in $\mathbb{C}^{n}$ and assume that $H_{1} \leq H_{2}$. Then

$$
H_{2}^{-1} \leq H_{1}^{-1} \quad \text { if and only if } \mathrm{i}\left(H_{1}\right)=\mathrm{i}\left(H_{2}\right) .
$$

Received by the editors May 13, 2011 and, in revised form, September 7, 2012.

2010 Mathematics Subject Classification. Primary 47A06, 47A63, 47B25; Secondary 15A09, $15 \mathrm{~A} 45,15 \mathrm{~B} 57$.

Key words and phrases. Selfadjoint operator, selfadjoint relation, inertia, matrix inequality, operator inequality, ordering.

This research was supported by grants from the Academy of Finland (project 139102) and the German Academic Exchange Service (DAAD), PPP Finland project 50740090.

The third author would like to thank the Deutsche Forschungsgemeinschaft (DFG) for the Mercator visiting professorship at the Technische Universität Berlin. 
The condition that the matrices $H_{1}$ and $H_{2}$ are invertible means that $i_{1}^{0}=i_{2}^{0}=0$; hence the condition $\mathrm{i}\left(H_{1}\right)=\mathrm{i}\left(H_{2}\right)$ in Theorem 1.1 is equivalent to $\mathrm{i}_{1}^{-}=\mathrm{i}_{2}^{-}$and to $\mathrm{i}_{1}^{+}=\mathrm{i}_{2}^{+}$. If in Theorem 1.1 the matrices $H_{1}$ and $H_{2}$ are not invertible, then the inverses $H_{1}^{-1}$ and $H_{2}^{-1}$ still exist in the sense of linear relations (multivalued mappings). With this interpretation Theorem 1.1 can be generalized to obtain the following two results, which are new and applicable already in the finite-dimensional setting (cf. [6, 7]).

Theorem 1.2. Let $H_{1}$ and $H_{2}$ be selfadjoint relations in $\mathbb{C}^{n}$ and assume that $H_{1} \leq H_{2}$. Then

$$
H_{2}^{-1} \leq H_{1}^{-1} \quad \text { if and only if } \mathrm{i}_{1}^{-}=\mathrm{i}_{2}^{-} .
$$

Theorem 1.3. Let $H_{1}$ and $H_{2}$ be selfadjoint relations in $\mathbb{C}^{n}$ and assume that $H_{1} \leq H_{2}$. Then

$$
-H_{1}^{-1} \leq-H_{2}^{-1} \quad \text { if and only if } \mathrm{i}_{1}^{-}+\mathrm{i}_{1}^{0}=\mathrm{i}_{2}^{-}+\mathrm{i}_{2}^{0} .
$$

Clearly, when the selfadjoint relations $H_{1}$ and $H_{2}$ are invertible matrices, then Theorem 1.2 and 1.3 coincide with Theorem 1.1 However, in the case of noninvertible matrices $H_{1}$ and $H_{2}$ the above statements are new extensions of Theorem 1.1. Note that since $H_{1}^{-1}$ and $H_{2}^{-1}$ are selfadjoint relations the condition $-H_{1}^{-1} \leq-H_{2}^{-1}$ is in general different from the condition $H_{2}^{-1} \leq H_{1}^{-1}$.

From either of the above theorems other previously known antitonicity results in the matrix literature can also be derived as special cases. For example the main antitonicity result for the Moore-Penrose inverse $H^{+}$of a selfadjoint matrix $H$ (see [4, Theorem 2]) can be obtained as a direct consequence of Theorem 1.2 .

Corollary 1.4. Let $H_{1}$ and $H_{2}$ be selfadjoint matrices in $\mathbb{C}^{n}$ and assume that $H_{1} \leq H_{2}$. Then

$$
H_{2}^{+} \leq H_{1}^{+} \quad \text { if and only if } \mathrm{i}\left(H_{1}\right)=\mathrm{i}\left(H_{2}\right) \text {. }
$$

It should be emphasized that both inequalities $H_{2}^{-1} \leq H_{1}^{-1}$ and $-H_{1}^{-1} \leq-H_{2}^{-1}$ occur naturally in the study of limits of monotone matrix functions and that they have different geometrical implications; see [7. Such inequalities between selfadjoint relations have interesting applications, for instance, in the area of differential equations: they appear in the study of the square-integrability of solutions of definite canonical systems of differential equations; see [6] and the references therein.

The objective of this paper is to prove antitonicity results analogous to Theorem 1.2 and Theorem 1.3 for selfadjoint operators or relations $H_{1}$ and $H_{2}$ in a separable, not necessarily finite-dimensional, Hilbert space. The results and their proofs can be read with the finite-dimensional case in mind. In fact, the proofs of the main two antitonicity theorems, Theorem 3.2 and Theorem 3.4 below, do not essentially simplify in the finite-dimensional setting. As a preparation some facts on selfadjoint relations in Hilbert spaces are in given Section 2. In particular, the notion of ordering for selfadjoint relations which are bounded from below and the concept of inertia are introduced. Section 3 contains the main results of the paper: the two infinite-dimensional variants of Theorem 1.2 and 1.3. The important ingredients in their proofs are an infinite-dimensional version of Theorem 1.1, which has been independently established in [9, 10, 19] (cf. [11, and see also [3]), combined 
with suitable perturbations arguments, and a general limit result on monotone operator functions. Various consequences of the two main antitonicity results are discussed, among them an infinite-dimensional version of the antitonicity result for Moore-Penrose inverses in Corollary [1.4.

\section{ORDERING AND INERTIA OF SELFADJOINT RELATIONS}

This section contains an introduction to selfadjoint relations in Hilbert spaces. In particular, the notions of ordering and inertia for selfadjoint relations in Hilbert spaces are introduced and investigated.

2.1. Linear relations. Let $\mathfrak{H}$ be a Hilbert space with scalar product $(\cdot, \cdot)$ and corresponding norm $\|\cdot\|$. A (closed) relation $H$ in $\mathfrak{H}$ is a (closed) linear subspace of the product space $\mathfrak{H} \times \mathfrak{H}$. As such, $H$ is considered to consist of pairs $\{h, k\} \in \mathfrak{H} \times \mathfrak{H}$ so that $H$ is the graph of a multivalued (linear) operator in $\mathfrak{H}$. The domain, range, kernel, and multivalued part of a relation $H$ are defined as follows:

$$
\begin{aligned}
\operatorname{dom} H=\{h \in \mathfrak{H}:\{h, k\} \in H\}, & \operatorname{ran} H=\{k \in \mathfrak{H}:\{h, k\} \in H\}, \\
\operatorname{ker} H=\{h \in \mathfrak{H}:\{h, 0\} \in H\}, & \operatorname{mul} H=\{k \in \mathfrak{H}:\{0, k\} \in H\} .
\end{aligned}
$$

Note that if $H$ is closed, then $\operatorname{ker} H$ and mul $H$ are closed subspaces. A number $\lambda \in \mathbb{C}$ is called an eigenvalue of $H$ if $\{h, \lambda h\} \in H$ for some nontrivial $h \in \mathfrak{H}$, which is then called an eigenvector. Similarly, $\infty$ is said to be an eigenvalue of $H$ if $\{0, k\} \in H$ or, equivalently, $k \in \operatorname{mul} H$, for some nontrivial $k \in \mathfrak{H}$, which is then called an eigenvector. The relation $H$ is an operator precisely when mul $H=\{0\}$, i.e., when $\infty$ is not an eigenvalue of $H$.

Each relation $H$ has an inverse $H^{-1}$ and an adjoint $H^{*}$, which are defined as

$$
\begin{aligned}
H^{-1} & =\{\{k, h\}:\{h, k\} \in H\}, \\
H^{*} & =\{\{h, k\} \in \mathfrak{H} \times \mathfrak{H}:(g, h)=(f, k) \text { for all }\{f, g\} \in H\} .
\end{aligned}
$$

In particular, dom $H^{-1}=\operatorname{ran} H$ and $\operatorname{ker} H^{-1}=\operatorname{mul} H$. Note that the adjoint is a closed relation in $\mathfrak{H}$ and that it coincides with the usual adjoint when $H$ is a densely defined operator.

For a relation $H$ in $\mathfrak{H}$ and $\lambda \in \mathbb{C}$, the relation $H-\lambda$ is given by

$$
H-\lambda=\{\{h, k-\lambda h\}:\{h, k\} \in H\} .
$$

Its inverse, $(H-\lambda)^{-1}$, is a relation whose kernel and multivalued part coincide with mul $H$ and $\operatorname{ker}(H-\lambda)$, respectively. Furthermore, it satisfies the following spectral mapping identity:

$$
(H-\lambda)^{-1}=-\frac{1}{\lambda}+\frac{1}{\lambda^{2}}\left(-H^{-1}-\left(-\frac{1}{\lambda}\right)\right)^{-1}, \quad \lambda \in \mathbb{C} \backslash\{0\} .
$$

For a closed relation $H$ the number $\lambda \in \mathbb{C}$ is said to belong to the resolvent set of $H, \lambda \in \rho(H)$, if $(H-\lambda)^{-1}$ is an everywhere defined operator. The resolvent set is an open subset of $\mathbb{C}$. For $\lambda \in \rho(H)$ the operator $(H-\lambda)^{-1}$ is called the resolvent operator of $H$ (at $\lambda)$. 
2.2. Selfadjoint relations. A relation $H$ is said to be symmetric if $(k, h) \in \mathbb{R}$ for all $\{h, k\} \in H$. By the polarization formula, $H$ is symmetric precisely when $H \subset H^{*}$. A relation $H$ is called selfadjoint if $H=H^{*}$; in particular, a selfadjoint relation is automatically closed. A selfadjoint relation $H$ in $\mathfrak{H}$ induces the following orthogonal decompositions of the space:

$$
\mathfrak{H}=\overline{\operatorname{dom}} H \oplus \operatorname{mul} H \quad \text { and } \quad \mathfrak{H}=\overline{\operatorname{ran}} H \oplus \operatorname{ker} H,
$$

where $\overline{\operatorname{dom}} H$ and $\overline{\text { ran }} H$ indicate the closures of dom $H$ and ran $H$, respectively. This shows that $H$ admits the following orthogonal decomposition:

$$
H=H_{s} \widehat{\oplus}(\{0\} \times \operatorname{mul} H),
$$

where $H_{s}=H \cap(\overline{\operatorname{dom}} H \times \overline{\operatorname{dom}} H)$, the so-called orthogonal operator part of $H$, is a selfadjoint operator in $\overline{\operatorname{dom}} H$ and $\{0\} \times \operatorname{mul} H$ is a selfadjoint relation in mul $H$. The symbol $\widehat{\oplus}$ in (2.3) indicates the orthogonality of the summands. It follows from (2.3) that the finite spectra of $H$ and of $H_{s}$ coincide. Hence $\mathbb{C} \backslash \mathbb{R} \subset \rho(H)$ and the selfadjoint operator part $H_{s}$ is bounded if and only if $\operatorname{dom} H$ is closed. Moreover, if ran $H$ is closed, then there exists a reduced neighborhood of 0 in $\mathbb{R}$ which belongs to $\rho(H)$, and 0 is at most an isolated eigenvalue of $H$. Let $E_{s}(\cdot)$ be the spectral function of $H_{s}$ in $\overline{\operatorname{dom}} H$. Define the spectral function $E(\cdot)$ for $H$ in $\mathfrak{H}$ by $E(t)=E_{s}(t) \oplus 0_{\text {mul } H}, t \in \mathbb{R},($ cf. (2.3) $)$ so that

$$
(H-\lambda)^{-1}=\int_{\mathbb{R}} \frac{1}{s-\lambda} d E(s), \quad \lambda \in \rho(H) .
$$

For a measurable function $\varphi: \mathbb{R} \rightarrow \mathbb{C}$, define $\varphi(H)=\varphi\left(H_{s}\right) \widehat{\oplus}(\{0\} \times \operatorname{mul} H)$.

A selfadjoint relation $H$ in a Hilbert space $\mathfrak{H}$ is said to be bounded from below by $m \in \mathbb{R}$ if its operator part $H_{s}$ is bounded from below by $m$ :

$$
\left(H_{s} h, h\right) \geq m(h, h) \text { for all } h \in \operatorname{dom} H=\operatorname{dom} H_{s} .
$$

Any such number $m$ is said to be a lower bound. The supremum of all lower bounds is called the lower bound of $H$. Any real number smaller than the lower bound belongs to $\rho(H)$. If the lower bound is nonnegative, then $H$ is called nonnegative: $H \geq 0$. Note that if $H$ has lower bound $m$, then $H-x$ has lower bound $m-x$ for any $x \in \mathbb{R}$. Therefore $H-x$ is nonnegative for all $x \leq m$. In particular, if $x<m$, then $(H-x)^{-1}$ is an everywhere defined positive bounded operator.

The square root $H^{1 / 2}$ of a nonnegative selfadjoint relation $H$ is defined as

$$
H^{1 / 2}=\left(H_{s}\right)^{1 / 2} \widehat{\oplus}(\{0\} \times \operatorname{mul} H) .
$$

For a nonnegative selfadjoint relation $H$ one has

$$
\operatorname{dom} H \subset \operatorname{dom} H^{1 / 2}, \quad \overline{\operatorname{dom}} H=\overline{\operatorname{dom}} H^{1 / 2}, \quad \operatorname{mul} H=\operatorname{mul} H^{1 / 2} .
$$

Clearly, if $H_{s}$ is bounded, then $\operatorname{dom} H=\operatorname{dom} H^{1 / 2}=(\operatorname{mul} H)^{\perp}$.

2.3. Ordering of selfadjoint relations. Let $H_{1}$ and $H_{2}$ be selfadjoint relations in a Hilbert space $\mathfrak{H}$ with lower bounds $m_{1}$ and $m_{2}$, respectively. Then $H_{1}$ and $H_{2}$ are said to satisfy $H_{1} \leq H_{2}$ if for a fixed $x<\min \left\{m_{1}, m_{2}\right\}$,

$$
0 \leq\left(\left(H_{2}-x\right)^{-1} h, h\right) \leq\left(\left(H_{1}-x\right)^{-1} h, h\right) \text { for all } h \in \mathfrak{H} ;
$$

see $[5,8,12$. The next proposition gives a characterization for the ordering of selfadjoint relations; see [8, 12. According to this proposition (2.6) holds automatically for all $x<\min \left\{m_{1}, m_{2}\right\}$ if it holds for some $x<\min \left\{m_{1}, m_{2}\right\}$. 
Proposition 2.1. Let $H_{1}$ and $H_{2}$ be selfadjoint relations in a Hilbert space $\mathfrak{H}$ with lower bounds $m_{1}$ and $m_{2}$, respectively. Then $H_{1}$ and $H_{2}$ satisfy $H_{1} \leq H_{2}$ if and only if for any $x<\min \left\{m_{1}, m_{2}\right\}$,

$$
\operatorname{dom}\left(H_{2}-x\right)^{1 / 2} \subset \operatorname{dom}\left(H_{1}-x\right)^{1 / 2}
$$

and

$$
\left\|\left(H_{1}-x\right)_{s}^{1 / 2} h\right\| \leq\left\|\left(H_{2}-x\right)_{s}^{1 / 2} h\right\| \text { for all } h \in \operatorname{dom}\left(H_{2}-x\right)^{1 / 2} .
$$

If dom $H_{1}$ and dom $H_{2}$ are closed or, equivalently, if the operator parts $\left(H_{1}\right)_{s}$ and $\left(H_{2}\right)_{s}$ are bounded, then by Proposition 2.1 (cf. (2.5) $) H_{1} \leq H_{2}$ if and only if (2.9) $\operatorname{dom} H_{2} \subset \operatorname{dom} H_{1} \quad$ and $\quad\left(\left(H_{1}\right)_{s} h, h\right) \leq\left(\left(H_{2}\right)_{s} h, h\right) \quad$ for all $h \in \operatorname{dom} H_{2}$.

In particular, if dom $H_{1}=\operatorname{dom} H_{2}=\mathfrak{H}$, i.e., if $H_{1}$ and $H_{2}$ are bounded selfadjoint operators, then the inequality $H_{1} \leq H_{2}$ has the usual meaning.

The inclusion (2.7) combined with (2.2) and (2.5) yields the following implication:

$$
H_{1} \leq H_{2} \quad \Rightarrow \quad \overline{\operatorname{dom}} H_{2} \subset \overline{\operatorname{dom}} H_{1} \quad \text { and } \quad \text { mul } H_{1} \subset \operatorname{mul} H_{2} .
$$

Corollary 2.2. Let $H_{1}$ and $H_{2}$ be selfadjoint relations in a Hilbert space $\mathfrak{H}$ with closed domains such that $H_{1} \leq H_{2}$. Then $-H_{2} \leq-H_{1}$ if and only if $\operatorname{dom} H_{1}=$ dom $H_{2}$ or, equivalently, mul $H_{1}=$ mul $H_{2}$.

Proof. By assumption the operator parts $\left(H_{1}\right)_{s}$ and $\left(H_{2}\right)_{s}$ are bounded, which guarantees that each of the relations $\pm H_{1}$ and $\pm H_{2}$ is bounded from below. Now, the implication $(\Rightarrow)$ is obtained by applying (2.10) to the inequalities $H_{1} \leq H_{2}$ and $-H_{2} \leq-H_{1}$. The implication $(\Leftarrow)$ follows directly from $(2.9)$.

Let $H_{j}$ be a selfadjoint relation in a Hilbert space $\mathfrak{H}$ with lower bound $m_{j}$ and let $E_{j}(\cdot)$ be its spectral function for $j=1,2$. Then for $x<m_{j}$,

$$
x\|h\|^{2}+\left\|\left(H_{j}-x\right)_{s}^{1 / 2} h\right\|^{2}=\int_{\mathbb{R}} s d\left(E_{j}(s) h, h\right), \quad h \in \operatorname{dom}\left(H_{j}-x\right)^{1 / 2} .
$$

Hence, the selfadjoint relations $H_{1}$ and $H_{2}$ satisfy $H_{1} \leq H_{2}$ if and only if the inclusion (2.7) and the following inequality are satisfied for any $x<\min \left\{m_{1}, m_{2}\right\}$ :

$$
\int_{\mathbb{R}} s d\left(E_{1}(s) h, h\right) \leq \int_{\mathbb{R}} s d\left(E_{2}(s) h, h\right) \quad \text { for all } \quad h \in \operatorname{dom}\left(H_{2}-x\right)^{1 / 2} .
$$

The next lemma will be useful in the proofs of Propositions 2.6 and 2.7 below.

Lemma 2.3. Let $H_{1}$ and $H_{2}$ be selfadjoint relations in a Hilbert space $\mathfrak{H}$ which are bounded from below and satisfy $H_{1} \leq H_{2}$. Let $E_{1}(\cdot)$ and $E_{2}(\cdot)$ denote the corresponding spectral measures. Then the following statements hold:

(i) $\operatorname{ran} E_{2}((-\infty, 0]) \cap \operatorname{ran}\left(I-E_{1}((-\infty, 0))\right) \subset \operatorname{ker} H_{1} \cap \operatorname{ker} H_{2}$;

(ii) $\operatorname{ran} E_{2}((-\infty, 0]) \cap \operatorname{ran}\left(I-E_{1}((-\infty, 0])\right)=\{0\}$;

(iii) $\operatorname{ran} E_{2}((-\infty, 0)) \cap \operatorname{ran}\left(I-E_{1}((-\infty, 0))\right)=\{0\}$.

Proof. Note first that since $\mathrm{H}_{2}$ is semibounded,

$$
\operatorname{ran} E_{2}((-\infty, 0]) \subset \operatorname{dom} H_{2} \subset \operatorname{dom}\left(H_{2}-x\right)^{1 / 2}, \quad x<\min \left\{m_{1}, m_{2}\right\} .
$$

Hence (2.11) holds for $h \in \operatorname{ran} E_{2}((-\infty, 0])$. 
(i) For $h \in \operatorname{ran} E_{2}((-\infty, 0]) \cap \operatorname{ran}\left(I-E_{1}((-\infty, 0))\right)$ the right-hand side of (2.11) is nonpositive and the left-hand side is nonnegative. Hence,

$$
\int_{\mathbb{R}} s d\left(E_{1}(s) h, h\right)=\int_{\mathbb{R}} s d\left(E_{2}(s) h, h\right)=0,
$$

and this implies (i).

(ii) Let $h \in \operatorname{ran} E_{2}((-\infty, 0]) \cap \operatorname{ran}\left(I-E_{1}((-\infty, 0])\right)$. If $h \neq 0$, the right-hand side of (2.11) is nonpositive and the left-hand side is positive. Hence $h=0$ and (ii) holds.

(iii) Let $h \in \operatorname{ran} E_{2}((-\infty, 0)) \cap \operatorname{ran}\left(I-E_{1}((-\infty, 0))\right)$. If $h \neq 0$, the right-hand side of (2.11) is negative and the left-hand side is nonnegative. Hence $h=0$ and (iii) holds.

The following result is included as preparation for Section 3

Lemma 2.4. Let $H$ be a selfadjoint relation in a Hilbert space $\mathfrak{H}$ and let $(\alpha, \beta)$ be a spectral gap of $H$, i.e., $(\alpha, \beta) \subset \rho(H)$. Then the following statements hold:

(i) The relations $(H-\alpha)^{-1}$ and $(H-\beta)^{-1}$ are selfadjoint with $-(H-\alpha)^{-1}$ and $(H-\beta)^{-1}$ being bounded from below. They are limits of $(H-t)^{-1}$ as $t \downarrow \alpha$ and $t \uparrow \beta$ respectively:

$$
(H-t)^{-1} \rightarrow(H-\alpha)^{-1}, \quad(H-t)^{-1} \rightarrow(H-\beta)^{-1},
$$

where the convergence is in the strong resolvent sense. Moreover, the inequalities

$$
-(H-t)^{-1} \leq-(H-\alpha)^{-1} \quad \text { and } \quad(H-t)^{-1} \leq(H-\beta)^{-1}
$$

hold for $\alpha<t<\beta$.

(ii) If $K_{\alpha}$ and $K_{\beta}$ are selfadjoint relations in $\mathfrak{H}$ with $-K_{\alpha}$ and $K_{\beta}$ being bounded from below, such that $-(H-t)^{-1} \leq-K_{\alpha}$ or $(H-t)^{-1} \leq K_{\beta}, \alpha<t<\beta$, then the limits $(H-\alpha)^{-1}$ and $(H-\beta)^{-1}$ satisfy

$$
-(H-\alpha)^{-1} \leq-K_{\alpha} \quad \text { or } \quad(H-\beta)^{-1} \leq K_{\beta} .
$$

Proof. The statements are proved for the right endpoint $\beta$; a similar reasoning applies to the left endpoint $\alpha$. Note first that if $E(\cdot)$ is the spectral function of $H$, then (2.4) shows that for all $t_{1}, t_{2} \in(\alpha, \beta)$ with $t_{1} \leq t_{2}$ and all $h \in \mathfrak{H}$,

$$
\left(\left(H-t_{2}\right)^{-1} h, h\right)-\left(\left(H-t_{1}\right)^{-1} h, h\right)=\int_{\mathbb{R} \backslash(\alpha, \beta)} \frac{t_{2}-t_{1}}{\left(s-t_{1}\right)\left(s-t_{2}\right)} d(E(s) h, h) .
$$

The support of the measure $d(E(\cdot) h, h)$ is contained in $\mathbb{R} \backslash(\alpha, \beta)$, and there the integrand is nonnegative. Hence, the operator function $(H-t)^{-1}$ is nondecreasing in $t \in(\alpha, \beta)$.

(i) Fix some $c \in(\alpha, \beta)$ and let $m_{c}$ be a lower bound for the bounded operator $(H-c)^{-1}$. As the function $t \mapsto(H-t)^{-1}$ is nondecreasing in $(\alpha, \beta)$, it follows that $m_{c}$ is a lower bound for $(H-t)^{-1}, t \in(c, \beta)$. Hence by [5, Theorem 3.5] there exists a selfadjoint relation $B$ in $\mathfrak{H}$, bounded from below by $m_{c}$, such that $(H-t)^{-1} \rightarrow B$ as $t \uparrow \beta$ in the strong resolvent sense or, equivalently, in the graph sense; cf. [5, Proposition 2.3] and [18]. Moreover, $(H-t)^{-1} \leq B$ holds for all $t \in(c, \beta)$. 
Hence, to prove (i) it suffices to verify $B=(H-\beta)^{-1}$. For this let $\{\phi, \psi\} \in B$. Since $B$ is the graph limit of $(H-t)^{-1}$ there exist $\left\{\phi_{t}, \psi_{t}\right\} \in(H-t)^{-1}$ with $\left\{\phi_{t}, \psi_{t}\right\} \rightarrow\{\phi, \psi\}$ as $t \uparrow \beta$. Since

$$
\left\{\psi_{t}, \phi_{t}+(t-\beta) \psi_{t}\right\} \in H-\beta \quad \text { and } \quad\left\{\phi_{t}+(t-\beta) \psi_{t}, \psi_{t}\right\} \in(H-\beta)^{-1},
$$

it follows that $\{\phi, \psi\} \in(H-\beta)^{-1}$, i.e., $B \subset(H-\beta)^{-1}$. Since both $B$ and $(H-\beta)^{-1}$ are selfadjoint, the equality $B=(H-\beta)^{-1}$ follows.

(ii) As $B=(H-\beta)^{-1}$ is bounded from below by $m_{c}$, the relation $(H-\beta)^{-1}-m_{c}$ is nonnegative. Recall that $\operatorname{dom}\left((H-\beta)^{-1}-m_{c}\right)^{1 / 2}=\mathfrak{H}_{0}$, where

$$
\mathfrak{H}_{0}=\left\{h \in \mathfrak{H}: \lim _{t \uparrow \beta}\left\|\left((H-t)^{-1}-m_{c}\right)^{1 / 2} h\right\|<\infty\right\} ;
$$

cf. [5, Theorem 3.5]. Now let $K_{\beta}$ be such that $(H-t)^{-1} \leq K_{\beta}$. Then by Proposition 2.1 for all $t \in(c, \beta)$,

$$
\operatorname{dom}\left(K_{\beta}-m_{c}\right)^{1 / 2} \subset \operatorname{dom}\left((H-t)^{-1}-m_{c}\right)^{1 / 2}
$$

and

$$
\left\|\left((H-t)^{-1}-m_{c}\right)^{1 / 2} h\right\| \leq\left\|\left(K_{\beta}-m_{c}\right)_{s}^{1 / 2} h\right\| \quad \text { for all } \quad h \in \operatorname{dom}\left(K_{\beta}-m_{c}\right)^{1 / 2} .
$$

Since $(H-t)^{-1}$ is a nondecreasing operator function on $(\alpha, \beta)$, the preceding inequality implies that

$$
\operatorname{dom}\left(K_{\beta}-m_{c}\right)^{1 / 2} \subset \mathfrak{H}_{0}=\operatorname{dom}\left((H-\beta)^{-1}-m_{c}\right)^{1 / 2} .
$$

Hence Proposition 2.1 yields $(H-\beta)^{-1} \leq K_{\beta}$.

2.4. Inertia of selfadjoint relations. The notion of inertia of a selfadjoint relation in a Hilbert space is defined by means of its associated spectral measure. In what follows the Hilbert space is assumed to be separable.

Definition 2.5. Let $H$ be a selfadjoint relation in a separable Hilbert space $\mathfrak{H}$ and let $E(\cdot)$ be the spectral measure of $H$. The inertia of $H$ is defined as the ordered quadruplet $\mathrm{i}(H)=\left\{\mathrm{i}^{+}(H), \mathrm{i}^{-}(H), \mathrm{i}^{0}(H), \mathrm{i}^{\infty}(H)\right\}$, where

$$
\begin{aligned}
\mathrm{i}^{+}(H) & =\operatorname{dim} \operatorname{ran} E((0, \infty)), & \mathrm{i}^{-}(H) & =\operatorname{dim} \operatorname{ran} E((-\infty, 0)), \\
\mathrm{i}^{0}(H) & =\operatorname{dim} \operatorname{ker} H, & \mathrm{i}^{\infty}(H) & =\operatorname{dim} \operatorname{mul} H .
\end{aligned}
$$

In particular, for a selfadjoint relation $H$ in $\mathbb{C}^{n}$, the quadruplet $\mathrm{i}(H)$ consists of the numbers of positive, negative, zero, and infinite eigenvalues of $H$; cf. [7. Hence, if $H$ is a selfadjoint matrix in $\mathbb{C}^{n}$, then $\mathrm{i}^{\infty}(H)=0$ and the remaining numbers make up the usual inertia of $H$; see, e.g. [13, 15] or the introduction.

The inertia numbers of a selfadjoint relation $H$ in a separable Hilbert space $\mathfrak{H}$ satisfy

$$
\mathrm{i}^{+}(H)+\mathrm{i}^{-}(H)+\mathrm{i}^{0}(H)+\mathrm{i}^{\infty}(H)=\operatorname{dim} \mathfrak{H} .
$$

Furthermore, the following identities hold:

$$
\begin{aligned}
\mathrm{i}\left(H^{-1}\right) & =\left\{\mathrm{i}^{+}(H), \mathrm{i}^{-}(H), \mathrm{i}^{\infty}(H), \mathrm{i}^{0}(H)\right\}, \\
\mathrm{i}\left(-H^{-1}\right) & =\left\{\mathrm{i}^{-}(H), \mathrm{i}^{+}(H), \mathrm{i}^{\infty}(H), \mathrm{i}^{0}(H)\right\} .
\end{aligned}
$$

The next proposition shows that the ordering of two selfadjoint relations in a separable Hilbert space implies certain inequalities between their inertia numbers; cf. [7, Proposition 3.6] for a finite-dimensional variant of Proposition 2.6. 
Proposition 2.6. Let $H_{1}$ and $H_{2}$ be selfadjoint relations in a separable Hilbert space $\mathfrak{H}$ which are bounded from below and satisfy $H_{1} \leq H_{2}$. Then their inertia $\mathrm{i}\left(H_{j}\right)=\left\{\mathrm{i}_{j}^{+}, \mathrm{i}_{j}^{-}, \mathrm{i}_{j}^{0}, \mathrm{i}_{j}^{\infty}\right\}, j=1,2$, satisfy the following inequalities:

(i) $\mathrm{i}_{1}^{\infty} \leq \mathrm{i}_{2}^{\infty}$ and $\mathrm{i}_{1}^{-}+\mathrm{i}_{1}^{0}+\mathrm{i}_{1}^{+} \geq \mathrm{i}_{2}^{-}+\mathrm{i}_{2}^{0}+\mathrm{i}_{2}^{+}$;

(ii) $\mathrm{i}_{1}^{-} \geq \mathrm{i}_{2}^{-}$and $\mathrm{i}_{1}^{-}+\mathrm{i}_{1}^{0} \geq \mathrm{i}_{2}^{-}+\mathrm{i}_{2}^{0}$;

(iii) $\mathrm{i}_{1}^{+}+\mathrm{i}_{1}^{\infty} \leq \mathrm{i}_{2}^{+}+\mathrm{i}_{2}^{\infty}$ and $\mathrm{i}_{1}^{0}+\mathrm{i}_{1}^{+}+\mathrm{i}_{1}^{\infty} \leq \mathrm{i}_{2}^{0}+\mathrm{i}_{2}^{+}+\mathrm{i}_{2}^{\infty}$.

Proof. (i) This is a direct consequence of the implication in (2.10).

(ii) If $\mathrm{i}_{1}^{-}=\infty$, then automatically $\mathrm{i}_{2}^{-} \leq \mathrm{i}_{1}^{-}$. Hence, in order to show $\mathrm{i}_{2}^{-} \leq \mathrm{i}_{1}^{-}$, assume that $\mathrm{i}_{1}^{-}<\infty$ and let $\mathcal{L}$ be a finite-dimensional subspace in $\operatorname{ran} E_{2}((-\infty, 0))$. Since $E_{1}((-\infty, 0))$ restricted to $\mathcal{L}$ is injective by Lemma 2.3 (iii), one has

$$
\operatorname{dim} \mathcal{L}=\operatorname{dim} E_{1}((-\infty, 0)) \mathfrak{L} \leq \operatorname{dim} \operatorname{ran} E_{1}((-\infty, 0))=\mathrm{i}_{1}^{-} .
$$

Thus any finite-dimensional subspace of $\operatorname{ran} E_{2}((-\infty, 0))$ has dimension at most $\mathrm{i}_{1}^{-}$, which implies that the space ran $E_{2}((-\infty, 0))$ itself has dimension at most $\mathrm{i}_{1}^{-}$, i.e. $\mathrm{i}_{2}^{-} \leq \mathrm{i}_{1}^{-}$.

The inequality $\mathrm{i}_{1}^{-}+\mathrm{i}_{1}^{0} \geq \mathrm{i}_{2}^{-}+\mathrm{i}_{2}^{0}$ can be shown in a similar way when (ii) in Lemma 2.3 is used instead of (iii).

(iii) By Lemma 2.3 (ii) the identity

$$
\operatorname{ran} E_{2}((-\infty, 0]) \cap\left(\operatorname{ran} E_{1}((0, \infty)) \oplus \operatorname{mul} H_{1}\right)=\{0\}
$$

holds. If $\mathrm{i}_{2}^{+}+\mathrm{i}_{2}^{\infty}=\infty$, then automatically $\mathrm{i}_{1}^{+}+\mathrm{i}_{1}^{\infty} \leq \mathrm{i}_{2}^{+}+\mathrm{i}_{2}^{\infty}$. Hence, in order to show $\mathrm{i}_{1}^{+}+\mathrm{i}_{1}^{\infty} \leq \mathrm{i}_{2}^{+}+\mathrm{i}_{2}^{\infty}$, assume that $\mathrm{i}_{2}^{+}+\mathrm{i}_{2}^{\infty}<\infty$ and let $\mathcal{L}$ be a finite-dimensional subspace in $\operatorname{ran} E_{1}((0, \infty)) \oplus \operatorname{mul} H_{1}$. Since $I-E_{2}((-\infty, 0])$ restricted to $\mathfrak{L}$ is injective by (2.14), one has

$$
\operatorname{dim} \mathcal{L}=\operatorname{dim}\left(I-E_{2}((-\infty, 0])\right) \mathfrak{L} \leq \operatorname{dim} \operatorname{ran}\left(I-E_{2}((-\infty, 0])\right)=\mathrm{i}_{2}^{+}+\mathrm{i}_{2}^{\infty} .
$$

Thus any finite-dimensional subspace of $\operatorname{ran} E_{1}((0, \infty)) \oplus$ mul $H_{1}$ has dimension at most $\mathrm{i}_{2}^{+}+\mathrm{i}_{2}^{\infty}$, which implies that the space ran $E_{1}((0, \infty)) \oplus$ mul $H_{1}$ itself has dimension at most $i_{2}^{+}+i_{2}^{\infty}$, i.e., $i_{1}^{+}+i_{1}^{\infty} \leq i_{2}^{+}+i_{2}^{\infty}$.

The inequality $\mathrm{i}_{1}^{0}+\mathrm{i}_{1}^{+}+\mathrm{i}_{1}^{\infty} \leq \mathrm{i}_{2}^{-}+\mathrm{i}_{2}^{+}+\mathrm{i}_{2}^{\infty}$ can be shown in a similar way when (iii) in Lemma 2.3 is used instead of (ii).

The case of equality in an inertia inequality of Proposition 2.6 has a specific geometric implication.

Proposition 2.7. Let $H_{1}$ and $H_{2}$ be selfadjoint relations in a separable Hilbert space $\mathfrak{H}$ which are bounded from below. Let $\mathrm{i}\left(H_{j}\right)=\left\{\mathrm{i}_{j}^{+}, \mathrm{i}_{j}^{-}, \mathrm{i}_{j}^{0}, \mathrm{i}_{j}^{\infty}\right\}$ be the inertia of $H_{j}, j=1,2$, and assume that $H_{1} \leq H_{2}$. Then the following statements hold:

(i) if $\mathrm{i}_{1}^{\infty}=\mathrm{i}_{2}^{\infty}<\infty$, then mul $H_{1}=\operatorname{mul} H_{2}$;

(ii) if $\mathrm{i}_{1}^{-}+\mathrm{i}_{1}^{0}=\mathrm{i}_{2}^{-}+\mathrm{i}_{2}^{0}<\infty$, then ker $H_{1} \subset \operatorname{ker} H_{2}$;

(iii) if $\mathrm{i}_{1}^{-}=\mathrm{i}_{2}^{-}<\infty$, then $\operatorname{ker} H_{2} \subset \operatorname{ker} H_{1}$.

In particular, if $\mathrm{i}_{1}^{-}=\mathrm{i}_{2}^{-}<\infty$ and $\mathrm{i}_{1}^{0}=\mathrm{i}_{2}^{0}<\infty$, then ker $H_{1}=\operatorname{ker} H_{2}$.

Proof. (i) This is a direct consequence of (2.10).

(ii) and (iii) Define the subspace $\mathfrak{L}_{0}=\operatorname{ran} E_{2}((-\infty, 0]) \cap \operatorname{ran}\left(I-E_{1}((-\infty, 0))\right)$. According to Lemma 2.3 (i) $\mathfrak{L}_{0} \subset \operatorname{ker} H_{1} \cap$ ker $H_{2}$. Furthermore, note that $\mathfrak{L}_{0}$ can be rewritten as

$$
\mathfrak{L}_{0}=\operatorname{ran} E_{2}((-\infty, 0]) \cap\left(\operatorname{ran} E_{1}((-\infty, 0))\right)^{\perp} .
$$


Since dim $\operatorname{ran} E_{2}((-\infty, 0])=\mathrm{i}_{2}^{-}+\mathrm{i}_{2}^{0}$ and $\operatorname{dim} \operatorname{ran} E_{1}((-\infty, 0))=\mathrm{i}_{1}^{-}<\infty$,

$$
\operatorname{dim} \mathfrak{L}_{0} \geq \mathrm{i}_{2}^{-}+\mathrm{i}_{2}^{0}-\mathrm{i}_{1}^{-} .
$$

In case (ii), the assumption together with (2.15) implies that $\operatorname{dim} \mathfrak{L}_{0} \geq \mathrm{i}_{1}^{0}=$ $\operatorname{dim}$ ker $H_{1}$. Combining this observation with the inclusion $\mathfrak{L}_{0} \subset \operatorname{ker} H_{1} \cap \operatorname{ker} H_{2} \subset$ ker $H_{1}$ yields that ker $H_{1} \cap \operatorname{ker} H_{2}=\operatorname{ker} H_{1}$ and, hence, that ker $H_{1} \subset \operatorname{ker} H_{2}$.

In case (iii), the assumption together with (2.15) implies that $\operatorname{dim} \mathfrak{L}_{0} \geq \mathrm{i}_{2}^{0}=$ $\operatorname{dim} \operatorname{ker} H_{2}$. Combining this observation with the inclusion $\mathfrak{L}_{0} \subset \operatorname{ker} H_{1} \cap \operatorname{ker} H_{2} \subset$ ker $H_{2}$ yields that ker $H_{1} \cap \operatorname{ker} H_{2}=\operatorname{ker} H_{2}$ and, hence, that ker $H_{2} \subset \operatorname{ker} H_{1}$.

\section{Antitonicity FOR SELFADJOINT RELATIONS}

The infinite-dimensional variants of the antitonicity theorems from the introduction are proved here by means of perturbation arguments, the spectral mapping result (2.1), and limit properties of monotone operator functions. Furthermore, various consequences and special cases of these results are also discussed.

\subsection{An antitonicity theorem for bounded and boundedly invertible op-} erators. The following theorem is the infinite-dimensional variant of Theorem 1.1 from the introduction. It was proved independently in [9, 10, 19, cf. [11. A simple proof is included here; it relies on the main arguments used in [10,11.

Theorem 3.1. Let $H_{1}$ and $H_{2}$ be bounded and boundedly invertible selfadjoint operators in a separable Hilbert space $\mathfrak{H}$. Let $\mathrm{i}\left(H_{j}\right)=\left\{\mathrm{i}_{j}^{+}, \mathrm{i}_{j}^{-}, \mathrm{i}_{j}^{0}, \mathrm{i}_{j}^{\infty}\right\}$ be the inertia of $H_{j}, j=1,2$, and assume that $\min \left\{\mathrm{i}_{2}^{+}, \mathrm{i}_{1}^{-}\right\}<\infty$ and that $H_{1} \leq H_{2}$. Then

$$
H_{2}^{-1} \leq H_{1}^{-1} \quad \text { if and only if } \mathrm{i}\left(H_{1}\right)=\mathrm{i}\left(H_{2}\right) \text {. }
$$

Proof. Observe that for the bounded and boundedly invertible selfadjoint operators $H_{1}$ and $H_{2}$ one has $\mathrm{i}_{j}^{0}=0=\mathrm{i}_{j}^{\infty}, j=1,2$. Hence $\mathrm{i}\left(H_{1}\right)=\mathrm{i}\left(H_{2}\right)$ is equivalent to $\mathrm{i}_{1}^{-}=\mathrm{i}_{2}^{-}$and $\mathrm{i}_{1}^{+}=\mathrm{i}_{2}^{+}$. Furthermore, observe that $\mathrm{i}_{1}^{-}<\infty$ implies that $\mathrm{i}_{2}^{-}<\infty$ and that $\mathrm{i}_{2}^{+}<\infty$ implies that $\mathrm{i}_{1}^{+}<\infty$; cf. Proposition 2.6.

$(\Rightarrow)$ In view of $(2.13)$ the equalities $\mathrm{i}_{1}^{-}=\mathrm{i}_{2}^{-}$and $\mathrm{i}_{1}^{+}=\mathrm{i}_{2}^{+}$follow by applying Proposition 2.6 to $H_{1} \leq H_{2}$ and $H_{2}^{-1} \leq H_{1}^{-1}$.

$(\Leftarrow)$ Assume that $\mathrm{i}\left(H_{1}\right)=\mathrm{i}\left(H_{2}\right)$ so that $\mathrm{i}_{1}^{-}=\mathrm{i}_{2}^{-}$and $\mathrm{i}_{1}^{+}=\mathrm{i}_{2}^{+}$. The asserted implication will be shown in two steps.

First consider the case that $\mathrm{i}_{1}^{-}<\infty$. Then $\mathrm{i}_{2}^{-}=\mathrm{i}_{1}^{-}<\infty$. Now define the operator $J$ as $I_{\mathrm{i}_{1}^{+}} \oplus-I_{\mathrm{i}_{1}^{-}}$. Then a result of G. Köthe (cf. [14, Satz 1.2]) shows the existence of bounded and boundedly invertible operators $V_{1}$ and $V_{2}$ such that

$$
H_{1}=V_{1}^{*} J V_{1} \text { and } H_{2}=V_{2}^{*} J V_{2} .
$$

By means of the above notation the inequality $H_{1} \leq H_{2}$ can be written as

$$
0 \leq J-U^{*} J U, \quad U=V_{1} V_{2}^{-1} .
$$

A simple calculation shows that

$$
\left(\begin{array}{cc}
I & 0 \\
J U^{*} & I
\end{array}\right)^{*}\left(\begin{array}{cc}
J-U J U^{*} & 0 \\
0 & J
\end{array}\right)\left(\begin{array}{cc}
I & 0 \\
J U^{*} & I
\end{array}\right)=\left(\begin{array}{cc}
I & J U \\
0 & I
\end{array}\right)^{*}\left(\begin{array}{cc}
J & 0 \\
0 & J-U^{*} J U
\end{array}\right)\left(\begin{array}{cc}
I & J U \\
0 & I
\end{array}\right) .
$$

Since congruence does not change the inertia of bounded operators, the inertia of the diagonal matrices in the above equation coincide, i.e.,

$$
\mathrm{i}^{-}\left(J-U J U^{*}\right)+\mathrm{i}^{-}(J)=\mathrm{i}^{-}(J)+\mathrm{i}^{-}\left(J-U^{*} J U\right) .
$$


As $\mathrm{i}^{-}(J)=\mathrm{i}_{1}^{-}<\infty$ and $\mathrm{i}^{-}\left(J-U^{*} J U\right)=0$ by (3.1) it follows that $\mathrm{i}^{-}\left(J-U J U^{*}\right)=0$, and hence $J-U J U^{*}$ is a nonnegative operator. Using the definition of $U$, this yields

$$
H_{2}^{-1}=\left(V_{2}^{*} J V_{2}\right)^{-1}=V_{2}^{-1} J V_{2}^{-*} \leq V_{1}^{-1} J V_{1}^{-*}=\left(V_{1}^{*} J V_{1}\right)^{-1}=H_{1}^{-1},
$$

which completes the proof in the case $\mathrm{i}_{1}^{-}<\infty$.

Next consider the case $\mathrm{i}_{2}^{+}<\infty$. Then it follows that $\mathrm{i}_{1}^{+}=\mathrm{i}_{2}^{+}<\infty$. By (2.13) this implies that $\mathrm{i}^{-}\left(-H_{1}\right)=\mathrm{i}^{-}\left(-H_{2}\right)<\infty$. Since $H_{1} \leq H_{2}$ is equivalent to $-H_{2} \leq-H_{1}$, the previous step shows that $-H_{1}^{-1} \leq-H_{2}^{-1}$, which is equivalent to $H_{2}^{-1} \leq H_{1}^{-1}$; see Corollary 2.2. This completes the proof of Theorem 3.1.

3.2. First main antitonicity theorem. The following theorem is the infinitedimensional version of Theorem 1.3 from the introduction. Recall that for selfadjoint relations $H_{1}$ and $H_{2}$ with closed ranges the operator parts of $H_{1}^{-1}$ and $H_{2}^{-1}$ are bounded; in particular, the relations $-H_{1}^{-1}$ and $-H_{2}^{-1}$ are bounded from below.

Theorem 3.2. Let $H_{1}$ and $H_{2}$ be selfadjoint relations in a separable Hilbert space $\mathfrak{H}$ which are bounded from below and have closed ranges. Let $\mathrm{i}\left(H_{j}\right)=\left\{\mathrm{i}_{j}^{+}, \mathrm{i}_{j}^{-}, \mathrm{i}_{j}^{0}, \mathrm{i}_{j}^{\infty}\right\}$ be the inertia of $H_{j}, j=1,2$, and assume that $\mathrm{i}_{1}^{-}+\mathrm{i}_{1}^{0}<\infty$ and that $H_{1} \leq H_{2}$. Then

$$
-H_{1}^{-1} \leq-H_{2}^{-1} \quad \text { if and only if } \mathrm{i}_{1}^{-}+\mathrm{i}_{1}^{0}=\mathrm{i}_{2}^{-}+\mathrm{i}_{2}^{0} \text {. }
$$

Proof. $(\Rightarrow)$ Apply Proposition 2.6 and (2.13) to the inequalities $H_{1} \leq H_{2}$ and $-H_{1}^{-1} \leq-H_{2}^{-1}$. Then the inertia equality $\mathrm{i}_{1}^{-}+\mathrm{i}_{1}^{0}=\mathrm{i}_{2}^{-}+\mathrm{i}_{2}^{0}$ follows.

$(\Leftarrow)$ Let $H_{1} \leq H_{2}$ and assume that $\mathrm{i}_{1}^{-}+\mathrm{i}_{1}^{0}=\mathrm{i}_{2}^{-}+\mathrm{i}_{2}^{0}<\infty$ holds. Since the ranges of $H_{1}$ and $H_{2}$ are closed, there exists a constant $\delta>0$, such that $(-\delta, \delta) \backslash\{0\} \subset \rho\left(H_{j}\right)$; i.e. $H_{j}$ has a spectral gap around 0 , and the point 0 is possibly an isolated eigenvalue of finite multiplicity, $j=1,2$. Define $\mu^{+}:=\min \{1, \delta\}$; then Proposition 2.1 implies that the inequality

$$
H_{1}-\epsilon_{1} \leq H_{2}-\epsilon_{2}, \quad 0<\epsilon_{2} \leq \epsilon_{1}<\mu^{+},
$$

holds. Clearly, $H_{j}\left(\epsilon_{j}\right):=H_{j}-\epsilon_{j}$ is boundedly invertible and its inertia is

$$
\mathrm{i}\left(H_{j}\left(\epsilon_{j}\right)\right)=\left\{\mathrm{i}_{j}^{+}\left(\epsilon_{j}\right), \mathrm{i}_{j}^{-}\left(\epsilon_{j}\right), \mathrm{i}_{j}^{0}\left(\epsilon_{j}\right), \mathrm{i}_{j}^{\infty}\left(\epsilon_{j}\right)\right\}=\left\{\mathrm{i}_{j}^{+}, \mathrm{i}_{j}^{-}+\mathrm{i}_{j}^{0}, 0, \mathrm{i}_{j}^{\infty}\right\}, \quad j=1,2 .
$$

Let $m_{j}$ be a lower bound for $H_{j}, j=1,2$. Then $m_{j}-1<m_{j}-\epsilon_{j}$ is a lower bound for $H_{j}\left(\epsilon_{j}\right), j=1,2$. Hence $H_{1}\left(\epsilon_{1}\right) \leq H_{2}\left(\epsilon_{2}\right)$ in (3.2) implies that

$$
0 \leq\left(H_{2}\left(\epsilon_{2}\right)-x\right)^{-1} \leq\left(H_{1}\left(\epsilon_{1}\right)-x\right)^{-1}, \quad x<\min \left\{0, m_{1}-1, m_{2}-1\right\} ;
$$

cf. (2.6). Using (2.1), this yields the inequality

$$
\left(-H_{2}\left(\epsilon_{2}\right)^{-1}+1 / x\right)^{-1} \leq\left(-H_{1}\left(\epsilon_{1}\right)^{-1}+1 / x\right)^{-1} .
$$

By (2.13) and (3.3) the inertia numbers of $-H_{j}\left(\epsilon_{j}\right)^{-1}, j=1,2$, are given by

$$
\mathrm{i}\left(-H_{j}\left(\epsilon_{j}\right)^{-1}\right)=\left\{\mathrm{i}_{j}^{-}\left(\epsilon_{j}\right), \mathrm{i}_{j}^{+}\left(\epsilon_{j}\right), \mathrm{i}_{j}^{\infty}\left(\epsilon_{j}\right), 0\right\}, \quad j=1,2 .
$$

Since $\left(0,-1 /\left(m_{j}-1\right)\right) \subset \rho\left(-H_{j}\left(\epsilon_{j}\right)^{-1}\right)$ the operator $-H_{j}\left(\epsilon_{j}\right)^{-1}+1 / x$ is bounded and boundedly invertible for all $x<\min \left\{0, m_{1}-1, m_{2}-1\right\}, j=1,2$. Hence (3.5) and (3.3) imply that for $j=1,2$ :

$$
\mathrm{i}\left(-H_{j}\left(\epsilon_{j}\right)^{-1}+1 / x\right)=\left\{\mathrm{i}_{j}^{-}\left(\epsilon_{j}\right), \mathrm{i}_{j}^{+}\left(\epsilon_{j}\right)+\mathrm{i}_{j}^{\infty}\left(\epsilon_{j}\right), 0,0\right\}=\left\{\mathrm{i}_{j}^{-}+\mathrm{i}_{j}^{0}, \mathrm{i}_{j}^{+}+\mathrm{i}_{j}^{\infty}, 0,0\right\} .
$$


Since by assumption $i_{1}^{-}+i_{1}^{0}=i_{2}^{-}+i_{2}^{0}<\infty$, Theorem 3.1 applied to (3.4) yields

$$
-H_{1}\left(\epsilon_{1}\right)^{-1}+1 / x \leq-H_{2}\left(\epsilon_{2}\right)^{-1}+1 / x, \quad 0<\epsilon_{2} \leq \epsilon_{1}<\mu^{+}
$$

or, equivalently,

$$
-\left(H_{1}-\epsilon_{1}\right)^{-1} \leq-\left(H_{2}-\epsilon_{2}\right)^{-1}, \quad 0<\epsilon_{2} \leq \epsilon_{1}<\mu^{+} .
$$

Now subsequently letting $\epsilon_{2} \downarrow 0$ and $\epsilon_{1} \downarrow 0$ in (3.6) in the strong resolvent sense and using Lemma 2.4 in each step, the inequality $-H_{1}^{-1} \leq-H_{2}^{-1}$ is obtained.

It is emphasized that the equivalence in Theorem 3.2 is not true without the minus signs; see Corollary 2.2.

Corollary 3.3. Let $H_{1}$ and $H_{2}$ be selfadjoint relations in a separable Hilbert space $\mathfrak{H}$ with closed domains and closed ranges. Let $\mathrm{i}\left(H_{j}\right)=\left\{\mathrm{i}_{j}^{+}, \mathrm{i}_{j}^{-}, \mathrm{i}_{j}^{0}, \mathrm{i}_{j}^{\infty}\right\}$ be the inertia of $H_{j}, j=1,2$, and assume that $\mathrm{i}_{1}^{-}+\mathrm{i}_{1}^{0}<\infty, \mathrm{i}_{2}^{\infty}<\infty$, and that $H_{1} \leq H_{2}$. Then the following statements are equivalent:

(i) $\mathrm{i}\left(H_{1}\right)=\mathrm{i}\left(H_{2}\right)$;

(ii) (a) $-H_{1}^{-1} \leq-H_{2}^{-1}$;

(b) $\mathrm{mul} H_{1}=$ mul $H_{2}$;

(c) $\operatorname{ker} H_{1}=\operatorname{ker} H_{2}$;

(iii) $-H_{2} \leq-H_{1},-H_{1}^{-1} \leq-H_{2}^{-1}$, and $H_{2}^{-1} \leq H_{1}^{-1}$.

Proof. (i) $\Rightarrow$ (ii) This follows from Theorem 3.2 and Proposition 2.7

(ii) $\Rightarrow$ (iii) Apply Corollary 2.2 to the inequalities $H_{1} \leq H_{2}$ and $-H_{1}^{-1} \leq-H_{2}^{-1}$. Then the desired inequalities follow.

(iii) $\Rightarrow$ (i) If the stated inequalities hold, then by Corollary $2.2 \mathrm{mul} H_{1}=\operatorname{mul~} H_{2}$ and $\operatorname{ker} H_{1}=\operatorname{ker} H_{2}$, i.e. $\mathrm{i}_{1}^{\infty}=\mathrm{i}_{2}^{\infty}$ and $\mathrm{i}_{1}^{0}=\mathrm{i}_{2}^{0}$. Furthermore, the inequality $-H_{1}^{-1} \leq-H_{2}^{-1}$ implies that $\mathrm{i}_{1}^{-}+\mathrm{i}_{1}^{0}=\mathrm{i}_{2}^{-}+\mathrm{i}_{2}^{0}$. Since $\mathrm{i}_{j}^{-}, \mathrm{i}_{j}^{0}$, and $\mathrm{i}_{j}^{\infty}$ are finite for $j=1,2$, (2.12) shows that (i) holds.

3.3. Second main antitonicity theorem. The following theorem is the infinitedimensional version of Theorem 1.2 from the introduction. It is emphasized that in contrast to Theorem 3.2 there is no closed range assumption on the relations. However, the conditions $H_{1} \leq H_{2}$ and $\mathrm{i}_{1}^{-}<\infty$ imply $\mathrm{i}_{2}^{-}<\infty$; hence $H_{1}^{-1}$ and $H_{2}^{-1}$ are both semibounded from below.

Theorem 3.4. Let $H_{1}$ and $H_{2}$ be selfadjoint relations in a separable Hilbert space $\mathfrak{H}$ which are bounded from below. Let $\mathrm{i}\left(H_{j}\right)=\left\{\mathrm{i}_{j}^{+}, \mathrm{i}_{j}^{-}, \mathrm{i}_{j}^{0}, \mathrm{i}_{j}^{\infty}\right\}$ be the inertia of $H_{j}$, $j=1,2$, and assume that $\mathrm{i}_{1}^{-}<\infty$ and that $H_{1} \leq H_{2}$. Then

$$
H_{2}^{-1} \leq H_{1}^{-1} \quad \text { if and only if } \mathrm{i}_{1}^{-}=\mathrm{i}_{2}^{-} \text {. }
$$

Proof. $\left(\Rightarrow\right.$ ) Apply Proposition 2.6 and (2.13) to $H_{1} \leq H_{2}$ and $H_{2}^{-1} \leq H_{1}^{-1}$. Then the inertia equality $\mathrm{i}_{1}^{-}=\mathrm{i}_{2}^{-}$follows.

$(\Leftarrow)$ Let $H_{1} \leq H_{2}$ and assume that $\mathrm{i}_{1}^{-}=\mathrm{i}_{2}^{-}<\infty$ holds. Then the negative spectrum of $H_{j}$ consists of $0 \leq \mathrm{i}_{j}^{-}<\infty$ eigenvalues (counting multiplicities), $j=$ 1,2. Let $\mu_{j}^{-}$be the largest negative eigenvalue of $H_{j}$ if $0<\mathrm{i}_{j}^{-}$and define

$$
\mu^{-}:= \begin{cases}\min \left\{1,-\mu_{1}^{-},-\mu_{2}^{-}\right\}, & \mathrm{i}_{1}^{-}=\mathrm{i}_{2}^{-}>0 \\ 1, & \mathrm{i}_{1}^{-}=\mathrm{i}_{2}^{-}=0 .\end{cases}
$$


Then

$$
H_{1}+\epsilon_{1} \leq H_{2}+\epsilon_{2}, \quad 0<\epsilon_{1} \leq \epsilon_{2}<\mu^{-},
$$

where $H_{j}+\epsilon_{j}$ is boundedly invertible and $\mathrm{i}\left(H_{j}+\epsilon_{j}\right)=\left\{\mathrm{i}_{j}^{+}+\mathrm{i}_{j}^{0}, \mathrm{i}_{j}^{-}, 0, \mathrm{i}_{j}^{\infty}\right\}, j=1,2$. Since by assumption $\mathrm{i}_{1}^{-}=\mathrm{i}_{2}^{-}<\infty$, Theorem 3.2 can be applied to (3.7) yielding

$$
-\left(H_{1}+\epsilon_{1}\right)^{-1} \leq-\left(H_{2}+\epsilon_{2}\right)^{-1}, \quad 0<\epsilon_{1} \leq \epsilon_{2}<\mu^{-} .
$$

Because $\left(H_{j}+\epsilon_{j}\right)^{-1}, j=1,2$, is a bounded operator, this inequality can be rewritten as

$$
\left(H_{2}+\epsilon_{2}\right)^{-1} \leq\left(H_{1}+\epsilon_{1}\right)^{-1}, \quad 0<\epsilon_{1} \leq \epsilon_{2}<\mu^{-} .
$$

Now subsequently letting $\epsilon_{1} \downarrow 0$ and $\epsilon_{2} \downarrow 0$ in (3.8) in the strong resolvent sense and using Lemma 2.4 in each step (which is possible since $\left(-\mu^{-}, 0\right) \subset \rho\left(H_{j}\right), j=1,2$ ), the inequality $H_{2}^{-1} \leq H_{1}^{-1}$ is obtained.

Theorem 3.4 with $\mathrm{i}_{1}^{-}=0$ implies the following well-known result for nonnegative selfadjoint operators and relations; cf. [2,8].

Corollary 3.5. Let $H_{1}$ and $H_{2}$ be selfadjoint relations in a separable Hilbert space H. Then

$$
0 \leq H_{1} \leq H_{2} \quad \text { if and only if } \quad 0 \leq H_{2}^{-1} \leq H_{1}^{-1} .
$$

The following corollary for (not necessarily bounded) selfadjoint operators extends Theorem 3.1] cf. [19, Theorems 1 and 2], [10, Theorem 2], and [11, Theorem 1.4].

Corollary 3.6. Let $H_{1}$ and $H_{2}$ be injective selfadjoint operators in a separable Hilbert space $\mathfrak{H}$ and let $\mathrm{i}\left(H_{j}\right)=\left\{\mathrm{i}_{j}^{+}, \mathrm{i}_{j}^{-}, \mathrm{i}_{j}^{0}, \mathrm{i}_{j}^{\infty}\right\}$ be the inertia of $H_{j}, j=1,2$. Then the following statements hold:

(i) if $H_{1}$ and $H_{2}$ are bounded from below, $\mathrm{i}_{1}^{-}<\infty$, and $H_{1} \leq H_{2}$, then

$$
H_{2}^{-1} \leq H_{1}^{-1} \quad \text { if and only if } \mathrm{i}\left(H_{1}\right)=\mathrm{i}\left(H_{2}\right) \text {; }
$$

(ii) if $-H_{1}$ and $-H_{2}$ are bounded from below, $\mathrm{i}_{2}^{+}<\infty$, and $-H_{2} \leq-H_{1}$, then

$$
-H_{1}^{-1} \leq-H_{2}^{-1} \quad \text { if and only if } \mathrm{i}\left(H_{1}\right)=\mathrm{i}\left(H_{2}\right) .
$$

Combining Theorem 3.2. Theorem 3.4, and Proposition 2.7 yields the following result.

Corollary 3.7. Let $H_{1}$ and $H_{2}$ be selfadjoint operators in a separable Hilbert space $\mathfrak{H}$ which are bounded from below and have closed ranges. Let $\mathrm{i}\left(H_{j}\right)=\left\{\mathrm{i}_{j}^{+}, \mathrm{i}_{j}^{-}, \mathrm{i}_{j}^{0}, \mathrm{i}_{j}^{\infty}\right\}$ be the inertia of $H_{j}, j=1,2$, and assume that $\mathrm{i}_{1}^{-}+\mathrm{i}_{1}^{0}<\infty$ and that $H_{1} \leq H_{2}$. Then

$$
H_{2}^{-1} \leq H_{1}^{-1}, \quad-H_{1}^{-1} \leq-H_{2}^{-1} \quad \text { if and only if } \mathrm{i}_{1}^{-}=\mathrm{i}_{2}^{-}, \quad \mathrm{i}_{1}^{0}=\mathrm{i}_{2}^{0} \text {, }
$$

in which case ker $H_{1}=\operatorname{ker} H_{2}$. 
3.4. An antitonicity theorem for Moore-Penrose inverses. We define the so-called Moore-Penrose inverse $H^{+}$of a selfadjoint operator $H$ in a Hilbert space as

$$
H^{+}:=P H^{-1} P,
$$

where $H^{-1}$ is the inverse of $H$ (in the sense of relations) and $P$ denotes the orthogonal projection onto $\overline{\text { ran }} H$ in $\mathfrak{H}$. It follows that

$$
H^{+}=\left(H^{-1}\right)_{s} \widehat{\oplus}(\operatorname{ker} H \times\{0\})
$$

holds. Note that the assumption mul $H=\{0\}$ implies $\operatorname{ker}\left(H^{-1}\right)_{s}=\operatorname{ker} H^{-1}=\{0\}$, and hence $\operatorname{ker} H^{+}=\operatorname{ker} H$ and $\mathrm{i}\left(H^{+}\right)=\mathrm{i}(H)$ hold.

The following theorem is the infinite-dimensional version of Corollary 1.4 from the introduction.

Theorem 3.8. Let $H_{1}$ and $H_{2}$ be selfadjoint operators in a separable Hilbert space $\mathfrak{H}$ which are bounded from below. Let $\mathrm{i}\left(H_{j}\right)=\left\{\mathrm{i}_{j}^{+}, \mathrm{i}_{j}^{-}, \mathrm{i}_{j}^{0}, \mathrm{i}_{j}^{\infty}\right\}$ be the inertia of $H_{j}$, $j=1,2$, and assume that $\mathrm{i}_{1}^{-}+\mathrm{i}_{1}^{0}<\infty$ and that $H_{1} \leq H_{2}$. Then

$$
H_{2}^{+} \leq H_{1}^{+} \quad \text { if and only if } \quad \operatorname{ker} H_{1}=\operatorname{ker} H_{2} \text { and } \mathrm{i}\left(H_{1}\right)=\mathrm{i}\left(H_{2}\right) \text {. }
$$

Proof. $(\Rightarrow)$ Since ker $H_{j}^{+}=\operatorname{ker} H_{j}, j=1,2$, it follows from Proposition 2.6 and (2.13) that $\mathrm{i}_{1}^{-}=\mathrm{i}_{2}^{-}<\infty$ and $\mathrm{i}_{1}^{0}=\mathrm{i}_{2}^{0}<\infty$ hold. Since $\mathrm{i}_{1}^{\infty}=\mathrm{i}_{2}^{\infty}=0$ by assumption, (2.12) implies that $\mathrm{i}_{1}^{+}=\mathrm{i}_{2}^{+}$and, therefore, $\mathrm{i}\left(H_{1}\right)=\mathrm{i}\left(H_{2}\right)$. The assertion ker $H_{1}=$ ker $\mathrm{H}_{2}$ follows from Proposition 2.7

$(\Leftarrow)$ The assumption $\mathrm{i}\left(H_{1}\right)=\mathrm{i}\left(H_{2}\right)$, together with Theorem 3.4. implies the inequalities $H_{2}^{-1} \leq H_{1}^{-1}$ and $\left(H_{2}^{-1}\right)_{s} \leq\left(H_{1}^{-1}\right)_{s}$. Therefore, as ker $H_{1}=\operatorname{ker} H_{2}$, it follows from (3.9) and Proposition 2.1 that $H_{2}^{+} \leq H_{1}^{+}$holds.

\section{REFERENCES}

[1] T. Ando, Löwner inequality of indefinite type, Linear Algebra Appl. 385 (2004), 73-80, DOI 10.1016/S0024-3795(03)00496-8. MR2063348(2005a:47026)

[2] Tsuyoshi Ando and Katsuyoshi Nishio, Positive selfadjoint extensions of positive symmetric operators, Tôhoku Math. J. (2) 22 (1970), 65-75. MR0264422 (41 \#9016)

[3] T. Ya. Azizov and V. L. Khatskevich, On selfadjoint operators associated with inequalities and applications to problems in mathematical physics (Russian, with Russian summary), Mat. Zametki 55 (1994), no. 6, 3-12, 157, DOI 10.1007/BF02110348; English transl., Math. Notes 55 (1994), no. 5-6, 549-554. MR1296007 (95i:47045)

[4] Jerzy K. Baksalary, Kenneth Nordström, and George P. H. Styan, Löwner-ordering antitonicity of generalized inverses of Hermitian matrices, Linear Algebra Appl. 127 (1990), 171-182, DOI 10.1016/0024-3795(90)90342-A. MR1048801 (91f:15014)

[5] J. Behrndt, S. Hassi, H.S.V. de Snoo, and H.L. Wietsma, Monotone convergence theorems for semi-bounded operators and forms with applications, Proc. Royal Soc. Edinburgh 140A (2010), no. 5, 927-951. MR2726115 (2012a:47052)

[6] Jussi Behrndt, Seppo Hassi, Henk de Snoo, and Rudi Wietsma, Square-integrable solutions and Weyl functions for singular canonical systems, Math. Nachr. 284 (2011), no. 11-12, 1334-1384, DOI 10.1002/mana.201000017. MR2832651 (2012h:34023)

[7] Jussi Behrndt, Seppo Hassi, Henk de Snoo, and Rudi Wietsma, Limit properties of monotone matrix functions, Linear Algebra Appl. 436 (2012), no. 5, 935-953, DOI 10.1016/j.laa.2011.05.024. MR2890898

[8] Earl A. Coddington and Hendrik S. V. de Snoo, Positive selfadjoint extensions of positive symmetric subspaces, Math. Z. 159 (1978), no. 3, 203-214. MR0500265 (58 \#17936)

[9] V. A. Derkach and M. M. Malamud, On a generalization of the Krěn-Stieltjes class of functions (Russian), Izv. Akad. Nauk Armenii Mat. 26 (1991), no. 2, 115-137; translation in J. Contemp. Math. Anal. 26 (1991), no. 2, 16-37. MR.1231843 (95c:47014) 
[10] S. Hassi and K. Nordström, Antitonicity of the inverse of selfadjoint operators, Schwerpunktprogramm der Deutschen Forschungsgemeinschaft, Forschungsbericht No. 364, 1992.

[11] Seppo Hassi and Kenneth Nordström, Antitonicity of the inverse and J-contractivity, (Timişoara, 1992), Oper. Theory Adv. Appl., vol. 61, Birkhäuser, Basel, 1993, pp. 149-161. MR $1246584(94 \mathrm{~m}: 47071)$

[12] S. Hassi, A. Sandovici, H. S. V. de Snoo, and H. Winkler, Form sums of nonnegative selfadjoint operators, Acta Math. Hungar. 111 (2006), no. 1-2, 81-105, DOI 10.1007/s10474006-0036-6. MR2188974(2007f:47022)

[13] Roger A. Horn and Charles R. Johnson, Matrix analysis, Cambridge University Press, Cambridge, 1985. MR832183 (87e:15001)

[14] Gottfried Köthe, Das Trägheitsgesetz der quadratischen Formen im Hilbertschen Raum, Math. Z. 41 (1936), no. 1, 137-152, DOI 10.1007/BF01180408. MR.1545607

[15] Peter Lancaster and Miron Tismenetsky, The theory of matrices, 2nd ed., Computer Science and Applied Mathematics, Academic Press Inc., Orlando, FL, 1985. MR792300 (87a:15001)

[16] Karl Löwner, Über monotone Matrixfunktionen, Math. Z. 38 (1934), no. 1, 177-216, DOI 10.1007/BF01170633. MR1545446

[17] Kenneth Nordström, Some further aspects of the Löwner-ordering antitonicity of the MoorePenrose inverse, Comm. Statist. Theory Methods 18 (1989), no. 12, 4471-4489 (1990), DOI 10.1080/03610928908830167. MR1046720 (91c:15002)

[18] Michael Reed and Barry Simon, Methods of modern mathematical physics. I, Functional analysis, 2nd ed., Academic Press Inc. [Harcourt Brace Jovanovich Publishers], New York, 1980. MR751959 (85e:46002)

[19] Yu. L. Shmul'yan, On inequalities between Hermitian operators (Russian), Mat. Zametki 49 (1991), no. 4, 138-141, 160, DOI 10.1007/BF01158223; English transl., Math. Notes 49 (1991), no. 3-4, 423-425. MR1119229 (92c:47017)

Institut für Numerische Mathematik, Technische Universität Graz, Steyrergasse 30, 8010 Graz, Austria

E-mail address: behrndt@tugraz.at

Department of Mathematics and Statistics, University of VaAsa, P.O. Box 700, Fi65101 VAASA, FinLAND

E-mail address: sha@uwasa.fi

Department of Mathematics and Statistics, University of VaAsa, P.O. Box 700, FI65101 VAASA, FinLAND

E-mail address: rwietsma@uwasa.fi

Johann Bernoulli Institute for Mathematics and Computer Science, University of Groningen, P.O. Box 407, 9700 AK Groningen, Netherlands

E-mail address: desnoo@math.rug.nl 